\title{
Analysis of Vibration of a Pipeline Supported on Elastic Soil Using Differential Transform Method
}

\author{
Mohammad Amin Rashidifar, ${ }^{1, *}$ Ali Amin Rashidifar ${ }^{2}$ \\ ${ }^{1}$ Department of Mechanical Engineering, Islamic Azad University, Shadegan Branch, Shadegan, Iran \\ ${ }^{2}$ Department of Computer Scince, Islamic Azad University, Shadegan Branch, Shadegan, Iran \\ *Corresponding author: rashidifar_58@y ahoo.com
}

Received April 6, 2013; Revised May 14, 2013; Accepted June 18, 2013

\begin{abstract}
In this paper, a simulation method called the Differential Transform Method (DTM) is employed to predict the vibration of an Euler-Bernoulli and Timoshenko beam (pipeline) resting on an elastic soil. The Differential Transform Method is introduced briefly. DTM can easily be applied to linear or nonlinear problems and reduces the size of computational work. With this method exact solutions may be obtained without any need of cumbersome work and it is a useful tool for analytical and numerical solutions. To make clear and illustrate the features and capabilities of the presented method, different problems have been solved by using the technique and solutions have been compared with those obtained in the literature.
\end{abstract}

Keywords: differential transform method, DTM, elastic soil, vibration, beam, pipeline

Cite This Article: Rashidifar, Mohammad Amin, Ali Amin Rashidifar, "Analysis of Vibration of a Pipeline Supported On Elastic Soil Using Differential Transform Method.” American Journal of Mechanical Engineering 1, no. 4 (2013): 96-102. doi: 10.12691/ajme-1-4-4.

\section{Introduction}

It is obvious that the analysis, design and performance of buried structures such as pipelines require an understanding of soil-structure interaction. Soil protects and supports the buried pipelines, thus reduces the risk of possible hazards that can lead to malfunction of the structure. Without utilizing the strength of the surrounding soil, buried pipelines usually cannot resist the loads and displacements to which they are subjected. Therefore, it is important to accurately evaluate soil restraint or loading on the buried pipelines so as to increase the service life of the structure.

In geotechnical engineering, there are numerous studies in which the structural elements as pipelines, shallow foundations and piles are presented as a beam. On the other hand various types of foundation models such as Winkler, Pasternak, Vlasov, etc. have been used in the analysis of structures on elastic foundations.

The well known and widely used mechanical model is the one devised by Winkler. According to the Winkler model, the beam-supporting soil is modeled as a series of closely spaced, mutually independent, linear elastic vertical springs which provide resistance in direct proportion to the deflection of the beam. In the Winkler model, the properties of the soil are described only by the parameter $\mathrm{k}$, which represents the stiffness of the vertical springs. However, due to its inability to take into account the continuity or cohesion of the soil, the Winkler model is considered as a rather crude approximation of the true mechanical behavior of the soil material. The assumption that there is no interaction between adjacent springs, also results in overlooking the influence of the soil on either side of the beam. To overcome this weakness, several two-parameter elastic foundation models have been suggested. In these models, while the first parameter represents the stiffness of the vertical spring as in the Winkler model, the second parameter is introduced to account for the coupling effect of the linear elastic springs [1].

As stated before pipelines, shallow foundations and piles may be modeled as a beam. There are different types of beam model. The well known's are Euler-Bernoulli and Timoshenko beam models. Euler-Bernoulli theory works well for slender beams. On the other hand this theory is not applicable for moderately short and thick beams. However, Timoshenko model evaluates the effects of transverse shear deformation and rotary inertia on the dynamic behavior of beams.

There are numerous studies on the vibration analysis of a beam resting on an elastic foundation. De Rosa [2] investigated the free vibration frequencies of Timoshenko beams on two-parameter elastic foundation, in which the differential equation of motion was deduced by following the geometrical approach. Matsunaga [3], based on the power series expansions of displacement components, derived a fundamental set of equations of a onedimensional higher order theory of deep elastic beamcolumns resting on elastic foundations by using Hamilton's principle. El-Mously [4] derived explicit formulae for the fundamental natural frequencies for vibration of finite Timoshenko-beams mounted on fin ite Pasternak-foundation by virtue of Rayleigh's principle. Chen [5] investigated the vibration of a beam resting on an 
elastic foundation by using differential quadrature element method (DQEM). Chen [6] developed the DQEM free vibration analysis model of non-prismatic shear deformable beams resting on the elastic foundation. Coşkun [7] studied the response of an elastic beam on a two-dimensional tensionless Pasternak foundation that was subjected to a central concentrated harmonic load, in which the governing differential equations of the problem were solved by using the trigonometric-hyperbolic functions and suitable boundary and contiunity conditions. Chen et al. [8] studied on a mixed method which combined the state space method and the differential quadrature method for bending and free vibration of arbitrarily thick beams resting on a Pasternak elastic foundation. Maheswari et al. [9] used finite difference method for the solution of governing differential equations of the problem investigating the response of a moving load on an infinite beam resting on a reinforced granular bed on soft soil. Auciello and De Rosa [10] used the differential quadrature method and the integral variational formulation Rayleigh - Ritz method for the dynamic analysis of a foundation beam on a two parameter elastic soil in the presence of subtangential follower force. Elfelsoufi and Azrar [11] presented a model for the investigation of buckling, flutter and vibration analyses of beams using the integral equation formulation. And recently Ruta [12] applied Chebyshev series approximation to solve the problem of nonprismatic Timoshenko beam resting on a two-parameter elastic foundation.

In this study, the natural frequencies of a pipeline represented by a uniform beam resting on a W inkler and Pasternak soil are investigated by the Differential Transform Method (DTM). The Differential Transform Method is a semi analytical-numerical technique based on the Taylor series expansion method for solving differential equations. It is different from the traditional high order Taylor series method. The Taylor series method is computationally taken long time for large orders. However, with DTM, doing some simple mathematical operations on differential equations a closed form series solution or an approximate solution can be obtained quickly. This method was first proposed by Zhou [13] in 1986 for solving both linear and nonlinear in itial-value problems of electrical circuits. Later, Chen and Ho [14] developed this method for partial differential equations and Ayaz $[15,16]$ studied two and three dimensional differential transform method of solution of the initial value problem for partial differential equations. Arikoğlu and Özkol [17] extended the differential transform method (DTM) to solve the integro-differential equations. Recently, the second author used the DTM method successfully to handle various kinds of rotating beam problems [18,19,20].

In order to show effectiveness of the DTM method, two different problems will be considered. The first one is Euler-Bernoulli beam resting on Winkler foundation. The second example is Timoshenko beam resting on Pasternak foundation. In the next section, the governing equations and the associated boundary conditions for the above mentioned problems will be given.

\section{The Equations of Motion}

Consider Euler-Bernoulli beam resting on Winkler foundation (Figure 1). The equation of motion for this problem is given as follows.

$$
E I \frac{\partial^{4} w}{\partial x^{4}}+k(x) w+\rho A(x)\left(\frac{\partial^{2} w}{\partial t^{2}}\right)=0
$$

where $\mathrm{k}$ is spring constant; $\mathrm{w}$ is deflection $(\mathrm{m})$; $\rho$ is the mass density $\left(\mathrm{kg} / \mathrm{m}^{3}\right)$; $\mathrm{A}$ is the cross sectional area $\left(\mathrm{m}^{2}\right)$; E is the Young's Modulus ( $\mathrm{Pa}$ ) and $\mathrm{I}$ is the area moment of inertia about the neutral axis $\left(\mathrm{m}^{4}\right)$. Here $\mathrm{x}$ is the horizontal space coordinate measured along the length of the beam and $\mathrm{t}$ is any particular instant of time.

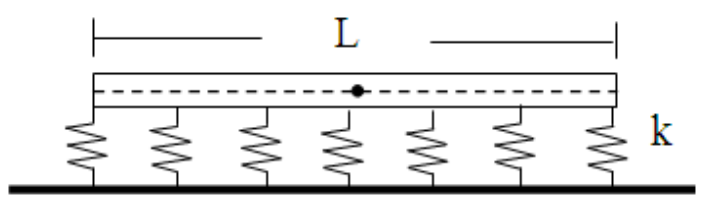

Figure 1. Geometry of a beam on Winkler foundation

In Figure 2, a Timoshenko beam on Pasternak foundation is shown. The governing equations for this problem are given as a system of differential equations. De Rosa [2] modeled the Pasternak foundation in two different forms. These forms lead the same differential equation for Euler-Bernoulli beam; on the other hand it is not true for Timoshenko beam which is adequate for stocky beams. Following De Rosa [2], we will call these definitions as Model I and Model II also. More information about these definitions can be found in De Rosa [2]. The first equation is the same for both Model I and Model II.

$$
k G A\left(\frac{\partial^{2} w}{\partial x^{2}}-\frac{\partial \phi}{\partial x}\right)-\rho A \frac{\partial^{2} w}{\partial t^{2}}-k_{w} w=0
$$

Where $\mathrm{G}$ is the shear modulus.

Model I:

$$
E I \frac{\partial^{2} \phi}{\partial x^{2}}+k G A\left(\frac{\partial w}{\partial x}-\phi\right)-\rho I \frac{\partial^{2} \phi}{\partial t^{2}}-k_{\phi} \phi=0
$$

Where $k_{\phi}$ is the constant of proportionality between bending moments and bending rotations.

Model II:

$$
E I \frac{\partial^{2} \phi}{\partial x^{2}}+k G A\left(\frac{\partial w}{\partial x}-\phi\right)-\rho I \frac{\partial^{2} \phi}{\partial t^{2}}-k_{w^{\prime}} \frac{\partial w}{\partial x}=0
$$

Where $k_{w^{\prime}}$ is the constant of proportionality between bending moments and global rotations.

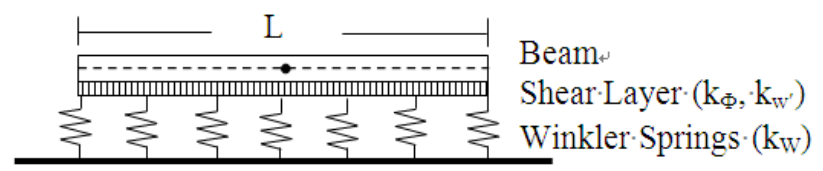

Figure 2. Geometry of a beam on Pasternak foundation

After completing equations of motion and associated boundary conditions, we will concentrate now about free vibration analysis of the beams resting on elastic foundations. 
In order to make free vibration analysis of the EulerBernoulli beam on the Winkler foundation, let us assume the solution is in the form of a sinusoidal variation of $\mathrm{w}(\mathrm{x}$, t) with circular frequency $\omega$ :

$$
w(x, t)=W(x) e^{i \omega t}
$$

Substituting equation (5) into equation (1), equations of motion is expressed as follows:

$$
E I \frac{d^{4} W}{d x^{4}}+k W=\rho A \omega^{2} W
$$

Similarly, for the free vibration analysis of Timoshenko beam resting on Pasternak foundation, the solution is assumed in the form of

$$
\begin{aligned}
& w(x, t)=W(x) e^{i \omega t} \\
& \phi(x, t)=\Phi(x) e^{i \omega t}
\end{aligned}
$$

Substituting equations (7) and (8) into equations (2) and (3) for Model I; and equations (4) and for Model II, the new form of equations of motion are expressed as follows:

$$
k G A\left(\frac{d^{2} W}{d x^{2}}-\frac{d \Phi}{d x}\right)+\rho A \omega^{2} W-k_{w} W=0
$$

Model I:

$$
E I \frac{d^{2} \Phi}{d x^{2}}+k G A\left(\frac{d W}{d x}-\Phi\right)+\rho I \omega^{2} \Phi-k_{\phi} \Phi=0
$$

Model II:

$$
E I \frac{d^{2} \Phi}{d x^{2}}+k G A\left(\frac{d W}{d x}-\Phi\right)+\rho I \omega^{2} \Phi-k_{w^{\prime}} \frac{d W}{d x}=0
$$

\section{Nondimensionalization}

The non-dimensional parameters for the Euler-beam on the Winkler foundation are defined as

$$
\xi=\frac{x}{L}, \bar{W}=\frac{W}{L}, \lambda=\frac{k L^{4}}{E I}, \bar{\omega}=\omega \sqrt{\frac{\rho A}{k}}
$$

Using these parameters, the nondimensional form of equation (6) can be written as:

$$
\frac{d^{4} \bar{W}}{d \xi^{4}}+\lambda\left(1-\bar{\omega}^{2}\right) \bar{W}=0
$$

Following De Rosa [2], the nondimensional parameters of Timoshenko beam on Pasternak foundation are given as follows:

$$
\begin{gathered}
\xi=\frac{x}{L}, \bar{W}=\frac{W}{L}, \alpha=\frac{k_{w} L^{4}}{E I}, S \phi=\frac{k_{\phi} L^{2}}{\pi^{2} E I}, S_{w^{\prime}}=\frac{k_{w^{\prime}} L^{2}}{\pi^{2} E I}(14) \\
c=\frac{k G}{E}, r=\sqrt{\frac{A L^{2}}{I}}, \chi=c r^{2}, \mu=\left(\frac{\rho A \omega^{2} L^{4}}{E I}\right)^{1 / 4}
\end{gathered}
$$

Using these parameters, nondimensional form of Equations can be written as follows:

$$
\chi \frac{d^{2} \bar{W}}{d \xi^{2}}-\chi \frac{d \Phi}{d \xi}+\left(\mu^{4}-\alpha\right) \bar{W}=0
$$

Model I:

$$
r^{2} \frac{d^{2} \Phi}{d \xi^{2}}+\chi r^{2} \frac{d \bar{W}}{d \xi}+\left(\mu^{4}-\chi r^{2}-\pi^{2} r^{2} s_{\phi}\right) \Phi=0
$$

Model II:

$r^{2} \frac{d^{2} \Phi}{d \xi^{2}}+\left(\chi r^{2}-\pi^{2} r^{2} s_{w^{\prime}}\right) \frac{d \bar{W}}{d \xi}+\left(\mu^{4}-\chi r^{2}\right) \Phi=0$

\section{Differential Transformation Method}

The differential transform method (DTM) is a transformation technique based on the Taylor series expansion and is a useful tool to obtain analytical solutions of the differential equations. In this method, certain transformation rules are applied and the governing differential equations and the boundary conditions of the system are transformed into a set of algebraic equations in terms of the differential transforms of the original functions and the solution of these algebraic equations gives the desired solution of the problem. It is different from high-order Taylor series method because Taylor series method requires symbolic computation is expensive for large orders. The DTM is an iterative procedure to obtain analytic Taylor Series solutions of differential equations.

Consider a function $f(x)$ which is analytic in a domain $\mathrm{D}$ and let $x=x_{0}$ represent any point in $\mathrm{D}$. The function $f(x)$ is then represented by a power series whose center is located at $x_{0}$. The differential transform of the function $f(x)$ is given by

$$
F(k)=\frac{1}{k !}\left(\frac{d^{k} f(x)}{d x^{k}}\right)_{x=x_{0}}
$$

where $f(x)$ is the original function and $F(k)$ is the transformed function.

The inverse transformation is defined as

$$
f(x)=\sum_{k=0}^{\infty}\left(x-x_{0}\right)^{k} F(k)
$$

Combining Eqs.(18) and (19) gives

$$
f(x)=\sum_{k=0}^{m} \frac{\left(x-x_{0}\right)^{k}}{k !}\left(\frac{d^{k} f(x)}{d x^{k}}\right)_{x=x_{0}}
$$

Considering Eq.(20), once more it is noticed that the concept of differential transform is derived from Taylor series expansion. However, the method does not evaluate the derivatives symbolically.

In actual applications, the function $f(x)$ is expressed by a finite series and Eq.(20) can be written as follows 


$$
f(x)=\sum_{k=0}^{m} \frac{\left(x-x_{0}\right)^{k}}{k !}\left(\frac{d^{k} f(x)}{d x^{k}}\right)_{x=x_{0}}
$$

which means that $f(x)=\sum_{k=m+1}^{\infty} \frac{\left(x-x_{0}\right)^{k}}{k !}\left(\frac{d^{k} f(x)}{d x^{k}}\right)_{x=x_{0}}$ is negligibly small. Here, the value of $m$ depends on the convergence rate of the natural frequencies. Theorems that are frequently used in the transformation of the differential equations and the boundary conditions are introduced in Table 1 and Table 2, respectively.

Table 1. DTM the orems use d for equations of motion

\begin{tabular}{|cc|}
\hline Original Function & Transformed Function \\
\hline$f(x)=g(x) \pm h(x)$ & $F(k)=G(k) \pm H(k)$ \\
$f(x)=\lambda g(x)$ & $F(k)=\lambda G(k)$ \\
$f(x)=g(x) h(x)$ & $F(k)=\sum_{l=0}^{k} G(l) H(k-l)$ \\
$f(x)=\frac{d^{n} g(x)}{d x^{n}}$ & $F(k)=\frac{(k+n) !}{k !} G(k+n)$ \\
$f(x)=x^{n}$ & $F(k)=\delta(k-n)=\left\{\begin{array}{lll}0 & \text { if } & k \neq n \\
1 & \text { if } & k=n\end{array}\right.$ \\
\hline
\end{tabular}

Table 2. DTM the orems use $d$ for boundary conditions

\begin{tabular}{|cccc|}
\hline \multicolumn{2}{c}{$x=0$} & \multicolumn{2}{c|}{$x=1$} \\
\hline Original B.C. & Transformed B.C & Original B.C. & Transformed B.C. \\
\hline$f(0)=0$ & $F(0)=0$ & $f(1)=0$ & $\sum_{k=0}^{\infty} F(k)=0$ \\
$\frac{d f}{d x}(0)=0$ & $F(1)=0$ & $\frac{d f}{d x}(1)=0$ & $\sum_{k=0}^{\infty} k F(k)=0$ \\
$\frac{d^{2} f}{d x^{2}}(0)=0$ & $F(2)=0$ & $\frac{d^{2} f}{d x^{2}}(1)=0$ & $\sum_{k=0}^{\infty} k(k-1) F(k)=0$ \\
$\frac{d^{3} f}{d x^{3}}(0)=0$ & $F(3)=0$ & $\frac{d^{3} f}{d x^{3}}(1)=0$ & $\sum_{k=0}^{\infty} k(k-1)(k-2) F(k)=0$ \\
\hline
\end{tabular}

\section{DTM Formulation and Solution Procedure}

First of all we will derive DTM form of Equation (9) which models Euler-beam on the Winkler foundation. Here we quit using the bar symbol on $\bar{W}$ and instead, we use $W$. If Table 1 is referred the following expression can be written easily.

$$
\begin{gathered}
(k+1)(k+2)(k+3)(k+4) W(k+4) \\
+\lambda\left(1-\bar{\omega}^{2}\right) W(k)=0
\end{gathered}
$$

If Eq. (21) is arranged, a simple requrrence relation can be obtained as follows:

$$
W(k+4)=\frac{\lambda\left(\bar{\omega}^{2}-1\right) W(k)}{(k+1)(k+2)(k+3)(k+4)}
$$

DTM formulation of which deals with Timoshenko beam resting on Pasternak foundation is derived as follows.

$$
\begin{aligned}
& \chi(k+2)(k+1) W(k+2)-\chi(k+1) \Phi(k+1) \\
& +\left(\mu^{4}-\alpha\right) W(k)=0 \\
& r^{2}(k+2)(k+1) \Phi(k+2)+\chi r^{2}(k+1) W(k+1) \\
& +\left(\mu^{4}-\chi r^{2}-\pi^{2} r^{2} s_{\phi}\right) \Phi(k)=0
\end{aligned}
$$

$$
\begin{aligned}
& r^{2}(k+2)(k+1) \Phi(k+2)+ \\
& \left(\chi r^{2}-\pi^{2} r^{2} s_{w^{\prime}}\right)(k+1) W(k+1)+\left(\mu^{4}-\chi r^{2}\right) \Phi(k)=0
\end{aligned}
$$$$
W(k+2)=-\frac{1}{\chi(k+2)(k+1)}\left[-\chi(k+1) \Phi(k+1)+\left(\mu^{4}-\alpha\right) W(k)\right]^{(27)}
$$$$
W(k+2)=-\frac{1}{\chi(k+2)(k+1)}\left[-\chi(k+1) \Phi(k+1)+\left(\mu^{4}-\alpha\right) W(k)\right]^{(28)}
$$$$
\begin{aligned}
\Phi(k+2)= & -\frac{1}{r^{2}(k+2)(k+1)} \\
& {\left[\left(\chi r^{2}-\pi^{2} r^{2} s_{w^{\prime}}\right)(k+1) W(k+1)+\left(\mu^{4}-\chi r^{2}\right) \Phi(k)\right] }
\end{aligned}
$$

Here Equation (27) denotes for Model I and Equation (28) denotes for Model II. 
The solution procedure of DTM will be shown for Model I with cantilever condition and the values are set to $\chi=40, r=12.5, \alpha=10, \mathrm{~S} \phi=1$.

$W(k)$ and $\Phi(k)$ values for $k=2,3,4 \ldots$ can now be evaluated in terms of $\mu, c_{1}$ and $c_{2}$. These values were achieved by using the Mathematica computer package.

$$
\begin{aligned}
& W(2)=-20 c_{1} \\
& W(3)=-0.00106667 c_{2}\left(-1542.13+\mu^{4}\right) \\
& W(4)=-0.000533333 c_{1}\left(31623.8-98.125 \mu^{4}\right) \\
& \Phi(2)=\frac{c_{2}}{2} \\
& \Phi(3)=-\frac{1}{240} c_{1}\left(1590+\mu^{4}\right) \\
& \Phi(4)=-\frac{1}{480} c_{2}\left(-202.392+0.628 \mu^{4}\right)
\end{aligned}
$$

where $c_{1}$ and $c_{2}$ are constants. Substituting all $W(i)$ and $\Phi(i)$ terms into boundary condition expressions, i.e. Eqs. (27) and (28), the following equation is obtained.

$$
A_{j 1}^{(n)}(\mu) c_{1}+A_{j 2}^{(n)}(\mu) c_{2}=0, j=1,2,3, . . n
$$

where $A_{j 1}^{(n)}(\mu), A_{j 2}^{(n)}(\mu)$ are polynomials of $\mu$ corresponding to $n^{\text {th }}$ term.

When Eq.(30) is written in matrix form, we get

$$
\left[\begin{array}{ll}
A_{11}^{n}(\mu) & A_{12}^{n}(\mu) \\
A_{21}^{n}(\mu) & A_{22}^{n}(\mu)
\end{array}\right]\left\{\begin{array}{l}
c_{1} \\
c_{2}
\end{array}\right\}=\left\{\begin{array}{l}
0 \\
0
\end{array}\right\}
$$

The eigenvalue equation is obtained from equation (31) as follows

$$
\left|\begin{array}{cc}
A_{11}^{n}(\mu) & A_{12}^{n}(\mu) \\
A_{21}^{n}(\mu) & A_{22}^{n}(\mu)
\end{array}\right|=0
$$

Solving equation (31), we get $\mu=\mu_{j}^{(n)}$ where $j=1,2,3, . . n$. Here, $\mu_{j}^{(n)}$ is the $j^{\text {th }}$ estimated eigenvalue corresponding to $n$. The value of $n$ is obtained by the following equation:

$$
\left|\mu_{j}^{(n)}-\mu_{j}^{(n-1)}\right| \leq \varepsilon
$$

where $\varepsilon$ is the tolerance parameter

If equation (37) is satisfied, then we have $j^{\text {th }}$ eigenvalue $\mu_{j}^{(n)}$. In general, $\mu_{j}^{(n)}$ are conjugated complex values, and can be written as $\mu_{j}^{(n)}=a_{j}+i b_{j}$. Neglecting the small imaginary part $b_{j}$, we have the $j^{\text {th }}$ natural frequency. In this study the value of $n=50$ was enough.

\section{Numerical Examples}

The computer package Mathematical is used to solve recurrence relations with associated boundary conditions. In the first example Euler beam resting on Winkler soil with different boundary conditions are investigated. Results are compared with Chen [5] study in which he used differential quadrature element method (DQEM).

\begin{tabular}{|c|c|c|c|c|c|}
\hline Method & & $\omega_{1}$ & $\omega_{2}$ & & $\omega_{3}$ \\
\hline DT M & & 9.92014 & 39.4911 & & 88.8321 \\
\hline DQEM & & 9.92014 & 39.4913 & & 89.4002 \\
\hline Exact Solution & & 9.92014 & 39.4911 & & 88.8321 \\
\hline Method & $\omega 1$ & $\omega 2$ & $\omega 3$ & $\omega 4$ & $\omega 5$ \\
\hline DTM & 22.3733 & 61.6728 & 120.903 & 199.859 & 298.556 \\
\hline DQEM & 22.3956 & 61.6811 & 120.910 & 199.885 & 298.675 \\
\hline
\end{tabular}
Chen [5] set all values to unity, such as $\mathrm{I}=\mathrm{E}=\mathrm{A}=\rho=1$, hence $\lambda=1$. In Table 3 , first three natural frequencies for simply supported beam are compared with Chen [5] results and exact solution. Very good agreement is observed with exact solution. The first five natural frequencies for fixed-fixed beam and cantilever beam are presented in Table 4 and Table 5 respectively. Again good a greement with Chen [5] results are demonstrated.

Table 3. Natural frequencies of a simply supported beam resting on Winkler foundation

Table 5. Natural frequencies of a can tile ver beam resting on Winkler foundation

\begin{tabular}{cccccc}
\hline Method & $\omega_{1}$ & $\omega_{2}$ & $\omega_{3}$ & $\omega_{4}$ & $\omega_{5}$ \\
\hline DTM & 3.65546 & 22.0572 & 61.7053 & 120.906 & 199.862 \\
DQEM & 3.65544 & 22.0572 & 61.7057 & 120.911 & 199.894 \\
\hline
\end{tabular}

In the second example, Timoshenko beam resting on beam is shown for $\alpha=100, \mathrm{r}=12.5$ and $\mathrm{S}_{\mathrm{w}^{\prime}}=\mathrm{S}_{\phi}=1$ and 2.5 . Pasternak foundation are investigated and results are compared with the ones De Rosa [2]. In Figure 3, the first nondimensional frequency $\mu$ vs. $\chi$, of a simply supported The difference between Model I and II is more evident for small $\chi$ values. For large $\chi$ values $\mu$ converges to EulerBernoulli limiting case. The same curves are given for a 
cantilever beam in Figure 4, but for different $\alpha$ value, which is now $\alpha=10$. Again the discrepancies are high for small $\chi$ values. In Table $6-9$ we presented nondimensional frequencies only limiting values for $\mathrm{K}_{\mathrm{TR}}$, such as $\mathrm{K}_{\mathrm{TR}}=0$ is cantilever beam case, on the other hand $\mathrm{K}_{\mathrm{TR}}=\infty$ is clamped-simply supported beam case. Results are given for first five natural frequencies, both for Model I and Model II. We could make comparisons only for first three modes of De Rosa [2]. Good agreement are shown between the results.

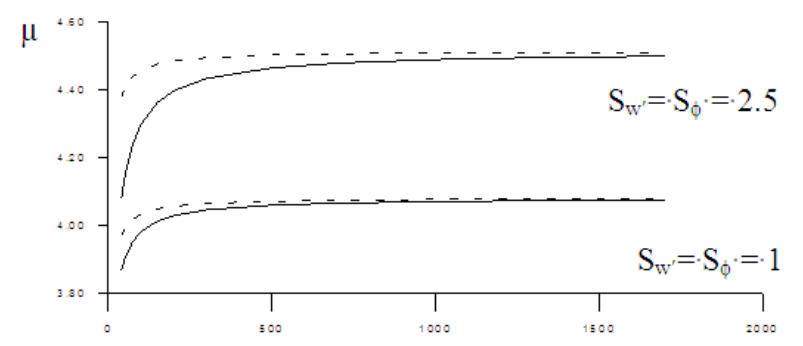

Figure 3. Simply supported beam with $\alpha=100$ and $r=12.5$. First nondimensional frequency as a function of $\chi$

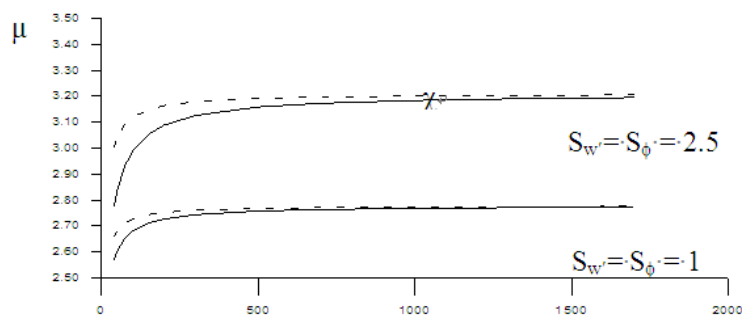

Figure 4. Cantilever beam with $\alpha=10$ and $r=12.5$. First nondimensional frequency as a function of $\chi$

Table 6. Clamped Simple Support $(\mathrm{KTR}=\infty)$ (Model I)

\begin{tabular}{cc}
\multicolumn{2}{c}{$\chi=40 \mathrm{r}=12.5 \alpha=10 \mathrm{~S} \phi=1$} \\
\hline Present & M. A. De Rosa \\
\hline 3.69645 & 3.6962 \\
5.54985 & 5.5495 \\
7.0857 & 7.0854 \\
8.39493 & ----- \\
9.50122 & ----- \\
\hline
\end{tabular}

Table 7. Clamped Simple Support $\left(\mathrm{K}_{\mathrm{TR}}=\infty\right)$ (Model II)

\begin{tabular}{cc}
\multicolumn{2}{c}{$\chi=40 \mathrm{r}=12.5 \alpha=10 \mathrm{~S}_{\mathrm{w}^{\prime}}=1$} \\
\hline Present & M. A. De Rosa \\
\hline 3.81887 & 3.8184 \\
5.67855 & 5.6782 \\
7.20297 & 7.2023 \\
8.49545 & ----- \\
9.04957 & ----- \\
\hline
\end{tabular}

Table 8. Cantile ver $\left(\mathrm{K}_{\mathrm{TR}}=\mathbf{0}\right)$ (Model $\left.\mathrm{I}\right)$

\begin{tabular}{cc}
\multicolumn{2}{c}{$\chi=40 \mathrm{r}=12.5 \alpha=10 \mathrm{~S} \phi=1$} \\
\hline Present & M. A. De Rosa \\
\hline 2.56732 & 2.5672 \\
4.40811 & 4.4079 \\
6.08403 & 6.0838 \\
7.49659 & ----- \\
8.70322 & ----- \\
\hline
\end{tabular}

Table 9. Cantile ver $\left(\mathrm{K}_{\mathrm{TR}}=\mathbf{0}\right)$ (Model II)

\begin{tabular}{cc}
\multicolumn{2}{c}{$\chi=40 \mathrm{r}=12.5 \alpha=10 \mathrm{~S}_{\mathbf{w}^{\prime}}=1$} \\
\hline Present & M. A. De Rosa \\
\hline 2.65852 & 2.6584 \\
4.55094 & 4.5507 \\
6.22309 & 6.2228 \\
7.6026 & ----- \\
8.73853 & ----- \\
\hline
\end{tabular}

\section{Conclusions}

In this study, we mainly concerned application of Differential Transformation Method (DTM) to predict the vibration of an Euler-Bernoulli and Timoshenko beam (pipeline) resting on an elastic soil. The study showed that by using DTM one can easily obtain solution for the problems described above. DTM eliminates cumbersome computational work needed by Taylor series. When the comparisons are made with the studies in literature, a very good agreement was observed.

\section{References}

[1] Avramidis IE, Morfidis K. 2006. 'Bending of beams on threeparameter elastic foundation. International Joumal of Solids and Structures’. volume 43. Page 357-375.

[2] De Rosa MA. 1995. 'Free vibration of Timoshenko beams on twoparameter elastic foundation'. Journal of Computers and Structures.volume 57(1). Page 151-156.

[3] Matsunaga H. 1999. 'vibration and bucklig of deep beam-columns on two-parameter elasti foundatins, Journal of Sound and Vibration'.volume 228(2). page 359-376.

[4] El-Mously M. 1999. 'Fundamental frequencies of Timoshenko beams mounted on pasternak foundation'. Journal of Sound and Vibration. Volume 228(2). Page 452-457.

[5] Chen CN. 2000. 'Vibration of prismatic beam on an elastic foundation by the differential quadrature element method'. Joumal of Computers and Structures. volume 77. Page 1-9.

[6] Chen CN. 2002. 'dqem vibration analyses of non-prismatic shear deformable beams resting on elastic foundations'. Journal of Sound and Vibration. Volume 255(5). Page 989-999.

[7] Coşkun İ. 2003. 'The response of a finite beam on a tensionless Pasternak foundation subjected to a harmonic load'. European Journal of Mechanics A/Solids. Volume 22. Page 151-161.

[8] Chen WQ, Lü CF, Bian ZG. 2004. 'A mixed method for bending and free vibration of beams resting on a Pasternak elastic foundation'. Applied Mathematical Modelling. volume28. Page 877-890.

[9] Maheshwari P, Chandra S, Basudhar PK. 2004. 'Response of beams on a tensionless extensible geosynthetic-reinforced earth bed subjected to moving loads'. Journal of Computers and Geotechnics. volume 31. Page 537-548.

[10] Auciello NM, De Rosa MA. 2004. 'Two approaches to the dynamic analysis of foundation beams subjected to subtangential forces'. Journal of Computers and Structures. Volume 82. Page 519-524.

[11] Elfelsoufi Z, Azrar L. 2005. 'Buckling, flutter and vibration analyses of beams by integral equation formulations. Computers and Structures'. Volume 83. Page 2632-2649.

[12] Ruta P. 2006. 'The application of Chebyshev polynomials to the solution of the nonprismatic Timoshenko beam vibration problem'. Journal of Sound and Vibration. Volume 296. Page 243-263.

[13] Zhou JK. 1986. 'Differential Transformation and its Application for Electrical Circuits'. China: Wuhan, Huazhong University Press

[14] Chen CK, Ho SH. 1999. 'Solving partial differential equations by two-dimensional differential transform method. Applied Mathematics and Comput at ion'. Volume 106. Page 171-179.

[15] Ayaz F. 2003. 'On the two-dimensional differential transform method'. Journal of Applied Mathematics and Computation 2003; 143: $361-374$ 
[16] Ayaz F. 2004. 'Solutions of the system of differential equations by differential transform method'. Journal of Applied Mathematics and Computation. Volume 147. Page 547-567.

[17] Arıkoğlu A, Özkol İ. 2005. 'Solution of boundary value problems for integro-differential equations by using differential transforms method'. Journal of Applied Mathematics and Computation. Volume 168. Page 1145-1158.

[18] Özdemir Ö, Kaya MO. 2006. 'Flapwise bending vibration analysis of a rotating tapered cantilever Bemoulli-Euler beam by differential transform method'. Journal of Sound and Vibration. Volume 289. Page 413-420.

[19] Kaya MO. 2006. 'Free Vibration Analysis of Rotating Timoshenko Beam by Differential Transform Method'. Journal of Aircraft Eng Aerosp Tec. Volume 78(3). Page 194-203.

[20] Özdemir Ö, Kaya MO. 2006. 'Flexural Vibration Analysis of Double Tapered Rotating Euler-Bernoulli Beam by Using the DifferentialTransform Method'. Joumal of Meccanica. 